\title{
The sensitivity, specificity, predictive values, and likelihood ratios of fecal occult blood test for the detection of colorectal cancer in hospital settings
}

This article was published in the following Dove Press journal:

Clinical and Experimental Gastroenterology

9 September 2015

Number of times this article has been viewed

\author{
Salah H Elsafil \\ Norah I Alqahtani' \\ Nawaf Y Zakary ${ }^{2}$ \\ Eidan MAl Zahrani ${ }^{3}$ \\ 'Clinical Laboratory Science \\ Department, Prince Sultan Military \\ College of Health Sciences, Dhahran, \\ ${ }^{2}$ Gastroenterology and Endoscopy \\ Unit, Internal Medicine Department, \\ King Fahd Military Medical Complex, \\ Dhahran, ${ }^{3}$ Prince Sultan Military \\ College of Heath Sciences, Dhahran, \\ Kingdom of Saudi Arabia
}

Objectives: To study the performance of a single test using two fecal occult blood tests with colonoscopy for the detection of colorectal cancer (CRC) for the first time in Saudi Arabia to determine possible implications for the anticipated colorectal screening program.

Materials and methods: We compared the performance of guaiac and immunochemical fecal occult blood tests for the detection of CRC among patients of 50-74 years old attending two hospitals in the Eastern Region of Saudi Arabia. Samples of feces were collected from 257 asymptomatic patients and 20 cases of confirmed CRC, and they were tested simultaneously by the guaiac-based occult blood test and monoclonal antibody-based immunoassay kit. Colonoscopy was performed on all participants and the results were statistically analyzed with both positive and negative occult blood tests of both methods.

Results: Of the 277 subjects, 79 tested positive for occult blood with at least one method. Overall, the number of those with an occult blood-positive result by both tests was $39(14.1 \%)$, while for $198(71.5 \%)$, both tests were negative $(P<0.0001)$; $40(14.4 \%)$ samples showed a discrepant result. Colonoscopy data were obtained for all 277 patients. A total of three invasive cancers were detected among the screening group. Of the three, the guaiac test detected two cases, while the immunochemical test detected three of them. Of the 20 control cases, the guaiac test detected $13 \mathrm{CRC}$ cases $(P=0.03)$, while the immunochemical test detected 16 of them $(P<0.0001)$. The sensitivity of guaiac and immunochemical tests for the detection of CRC in the screening group was $50.00 \%(95 \%$ confidence interval $[\mathrm{CI}]=6.76-93.24)$ and $75.00 \%(95 \%$ $\mathrm{CI}=19.41-99.37)$, respectively. For comparison, the sensitivity of the guaiac fecal occult blood test for detecting CRC among the control group was $65.00 \%(95 \% \mathrm{CI}=40.78-84.61)$ while that of FIT was $80.00 \%(95 \% \mathrm{CI}=56.34-94.27)$. The specificity of the guaiac and immunoassay tests was $77.87 \%(95 \% \mathrm{CI}=72.24-82.83)$ and $90.12 \%(95 \% \mathrm{CI}=85.76-93.50)$, respectively. The positive likelihood ratio of guaiac and immunochemical tests for the detection of CRC was $2.26(95 \% \mathrm{CI}=0.83-6.18)$ and $7.59(95 \% \mathrm{CI}=3.86-14.94)$, whereas the negative likelihood ratio was $0.64(95 \% \mathrm{CI}=0.24-1.71)$ and $0.28(95 \% \mathrm{CI}=0.05-1.52)$, respectively. The positive predictive values of guaiac and immunochemical tests were $3.45 \%(95 \% \mathrm{CI}=0.426-11.91)$ and $10.71 \%$ (95\% CI $=2.27-28.23$ ), respectively. There was no marked difference in the negative predictive values for both methods. The sensitivity of the fecal occult blood test by FIT was significantly higher for stages III and IV colorectal cancer than for stages I and II $(P=0.01)$ and it was insignificant for the guaiac fecal occult blood test $(P=0.07)$.

Conclusion: In areas where other advance screening methods of CRC are not feasible, the use of FIT can be considered.

Keywords: fecal occult blood test, guaiac, immunochemical, endoscopy, colorectal cancer 


\section{Introduction}

Colorectal cancer (CRC) is an important health concern and a leading cause of death among adults worldwide. In Saudi Arabia, a total of 4,201 cases of CRC were registered in the national Saudi Cancer Registry, with a noticeable increase in incidence rates between 2001 and 2006. ${ }^{1}$ This cancer ranked first among the male population and third among the female population with an overall age-standardized incidence rate of 6.6 per 1,000,000. ${ }^{2}$ Between 1994 and 2003, age-standardized rates for CRC in Saudi Arabia had increased almost twofold. ${ }^{3}$

Early detection of CRC is one of the best approaches to reduce related deaths. A broad spectrum of choices is available for CRC screening, including fecal occult blood (FOB) testing (FOBT), flexible sigmoidoscopy, and colonoscopy. ${ }^{4,5}$

FOBT detects blood in the stool that is not visible on gross inspection, usually less than $50 \mathrm{mg}$ of hemoglobin per gram of stool. ${ }^{6}$ The test is intended for the determination of gastrointestinal bleeding found in a number of gastrointestinal disorders including diverticulitis, colitis, polyps, and CRC. Three randomized controlled clinical trials showed that FOBT reduced the risk for death from CRC. ${ }^{7-9}$

Two types of FOBTs of different analytical principles are available: the traditional guaiac (G)-FOBT; and the antibodybased fecal immunochemical testT(FIT). The G-FOBT is based on the oxidation of phenolic compounds present in the guaiac (ie, guaiaconic acids), impregnated on the card that detects the pseudoperoxidase activity of the hematin portion of any hemoglobin, resulting in the production of a blue color. ${ }^{10,11}$ G-FOBTs are not specific for human hemoglobin and they detect any peroxidase found in feces (eg, plant peroxidases, heme in red meat), and they are affected by certain chemicals (eg, vitamin C). ${ }^{12}$ It may also detect bleeding from any site in the gastrointestinal tract, including the stomach. ${ }^{13}$

Recently, an immunoassay for the FOBT has been introduced utilizing two monoclonal antibodies that specifically detect the presence of human globin in feces and is thus more specific for bleeding from the distal gut.

In the early 1970 s, G-FOBTs were first proposed for the screening of $\mathrm{CRC},{ }^{14}$ and evidence has shown that they reduce both the incidence and mortality of CRC, ${ }^{7-9,15,16}$ although positive and negative predictive values were suboptimal. ${ }^{17} \mathrm{CRC}$ screening by G-FOBTs had also been complicated by a high incidence of false-positive results, especially when patients do not follow a restricted diet before testing. ${ }^{11}$

More recently, FIT has been widely used as an alternative to G-FOBT for CRC screening programs. Several methods of FIT exist including automated quantitative analysis.
Comparisons of different techniques to detect occult blood in the stool have been widely performed. Recent studies that compared G-FOBT and FIT in screening populations indicated superiority of the latter for the detection of both cancers and advanced adenomas. ${ }^{17-19}$ In the past few years, CRC screening has become more popular and colonoscopy has been postulated as the gold standard. ${ }^{20}$

Although extensive literature concerning FOBT and CRC screening is available, these are mostly community based with only a few being hospital based. While community-based screening studies provide critical information on program sensitivity and the acceptability of a test in a large population, often only small numbers of CRCs are detected. ${ }^{21}$ In referral hospital-based studies, a higher prevalence of $\mathrm{CRC}$ will allow for better understanding of the performance of FOBTs for the detection of CRC as compared to colonoscopy. Most other study designs have included colonoscopy for positive FOBTs only. ${ }^{17-19,22}$ In order to directly measure the specificity of the FOBTs, the colonoscopy results of individuals with negative results should also be available. To allow for the better evaluation of the methods of FOBTs, a comparative study design that includes the performance of both tests in parallel on the same stool samples is needed..$^{23}$ The objective of this study is to compare the sensitivity, specificity, positive predictive value, and negative predictive value of G-FOBT and FIT in the same stool samples among patients attending hospitals who all underwent colonoscopy. To the best of our knowledge, no previous work has been done in Saudi Arabia for the detection of CRC by FOBT.

\section{Materials and methods Study design}

The Research and Ethics Committee at the King Fahd Military Medical Complex (Dhahran, Saudi Arabia) approved this prospective cohort study protocol, including patients who reported to two tertiary hospitals in the eastern region of Saudi ArabiaKing Fahd Military Medical Complex (Dhahran, Saudi Arabia); and King Faisal Specialist Hospital (Dammam, Saudi Arabia) from June 2012 through May 2013.

All asymptomatic participants that reported to hospital in this study were 50-74 years of age; they were tested once for FOB by two simultaneous methods, and they were scheduled for colonoscopy. Patients who reported symptoms of disease of the lower gastrointestinal tract were excluded. The medical record number of each of the participants was recorded. The results of the participants who were offered colonoscopy were taken from the medical record during the final 6 months of the 
study period (ie, June 2013 through December 2013). Twenty confirmed CRC cases were recruited as a control.

\section{Fecal occult blood test}

During the study period, 277 samples of feces were collected and tested simultaneously using the guaiac-based ColoScreen $^{\circledR}$ by Helena Laboratories (Cat no: 5073; Beaumont, TX, USA) and the monoclonal antibody-based immunoassay (RAPEPKT313) kit by DIAsource $\odot$ (DIAsource ImmunoAssays SA, Nivelles, Belgium) using the 1-day method.

ColoScreen ${ }^{\circledR}$ was performed according to the manufacturer's instructions. In brief, using the applicator, very thin smears of stool from different sites were applied in boxes A and $\mathrm{B}$, and they were allowed to air dry before the cover was closed. The perforated window on the back of the slide was then opened and two drops of ColoScreen developer was applied to the back of boxes A and B before reading the results after 30 seconds and within 2 minutes. Any trace of blue color, within or on the outer rim of the specimen, was reported as positive for occult blood.

RAPEPKT313 was performed according to the manufacturer's instructions. In brief, a stool specimen is collected into the sampling tube containing extraction solution. After mixing the stool sample, a test strip is screwed into the sampling tube by breaking the bottom seal of the sampling tube while maintaining a vertical position, and it was allowed to settle for approximately 1 minute. The extracted fecal solution flows into the bottom space of the test strip and triggers the start of the FOB immunoassay. If human hemoglobin is present at a level higher than $50 \mathrm{ng} / \mathrm{mL}$ in a fecal sample extract, a red colored band appears in the test region, which is located in the lower half of the test membrane. A similar colored band must appear in the control region located in the upper-half of the test membrane, indicating that the test strip is functioning properly and the result is valid.

\section{Colonoscopy}

All participants underwent complete colonoscopy in one of the two hospitals. Colonoscopies were performed in a standard fashion by experienced gastroenterologists. The location and size of all polypoid lesions were recorded and the tumor specimens were pathologically classified as previously described. ${ }^{24}$

\section{Statistics analysis}

Statistical analysis was performed using SAS software version 9.1. Sensitivities, specificities, and predictive values of G-FOBT and FIT for CRC were calculated as previously described. ${ }^{6,25}$ We used the chi-squared and $t$-test, and $P$-values $>0.05$ were considered statistically significant.

\section{Results}

Overall, 277 individuals between 50 and 74 years of age (mean: 63.8 years; standard deviation $=7.9$ years) (189 males and 88 females) were tested by the two methods, G-FOBT and FIT.

Table 1 shows that the FOBT positivity rates of the guaiac and immunoassay tests for the screening and control groups were $22.6 \%, 12.1 \%, 65 \%$, and $80 \%$, respectively. For the occult blood tests, 39 (14.1\%) patients were positive for both tests, while 198 (71.5\%) were negative by both tests. Overall, $40(14.4 \%)$ samples showed a discrepant result.

Colonoscopy was successfully performed on all 257 patients in the screening group and three invasive cancers were detected. Of the patients endoscoped, 37 were occult blood-positive by both or at least one test, and the other 194 were negative by both methods. Among the 20 cancer cases in the control group, 13 and 16 tested positive by G-FOBT and FIT, respectively. Tables 2 and 3 indicated the performance characteristics of G-FOBT and FIT for the detection of $\mathrm{CRC}$, respectively.

The sensitivity of G-FOBT and FIT for the detection of CRC among the screening group was $50.00 \%$ (95\% confidence interval $[\mathrm{CI}]=6.76-93.24)$ and $75.00 \%(95 \%$ $\mathrm{CI}=19.41-99.37)$, respectively. The specificity of G-FOBT and FIT was $77.87 \%(95 \% \mathrm{CI}=72.24-82.83)$ and $90.12 \%$ $(95 \%$ CI $=85.76-93.50)$, respectively (Table 4$)$. The positive likelihood ratio of guaiac and immunochemical tests for the detection of $\mathrm{CRC}$ was $2.26(95 \% \mathrm{CI}=0.83-6.18)$ and 7.59 (95\% CI $=3.86-14.94)$, whereas the negative likelihood ratio was $0.64(95 \% \mathrm{CI}=0.24-1.71)$ and $0.28(95 \%$ $\mathrm{CI}=0.05-1.52$ ), respectively.

The positive predictive values of G-FOBT and FIT were $3.45 \%(95 \% \mathrm{CI}=0.426-11.91)$ and $10.71 \%(95 \%$ CI $=2.27-28.23)$, respectively. There was no marked

Table I Performance characteristics of FIT compared with G-FOBT among the screening and control groups

\begin{tabular}{|c|c|c|c|c|c|}
\hline \multirow[t]{3}{*}{ G-FOBT } & \multicolumn{4}{|l|}{ FIT } & \multirow[t]{3}{*}{ Total } \\
\hline & \multicolumn{2}{|c|}{ Screening group } & \multicolumn{2}{|c|}{ Control group } & \\
\hline & Positive & Negative & Positive & Negative & \\
\hline Positive & 26 & 32 & 13 & 0 & 71 \\
\hline Negative & 5 & 194 & 3 & 4 & 206 \\
\hline Total & 31 & 226 & 16 & 4 & 277 \\
\hline
\end{tabular}

Note: Chi-squared $=108.93 ; P<0.0001$.

Abbreviations: FIT, fecal immunochemical test; G-FOBT, guaiac fecal occult blood test. 
Table 2 Performance characteristics of G-FOBT for detecting colorectal cancer

\begin{tabular}{lllll}
\hline G-FOBT & \multicolumn{2}{l}{ Screening group } & Total & \multicolumn{2}{l}{ Control group } \\
\cline { 2 - 3 } & \multicolumn{2}{l}{ Colorectal cancer } & & Colorectal cancer \\
\cline { 2 - 3 } & Positive & Negative & & \\
\hline Positive & 2 & 56 & 58 & 13 \\
Negative & 2 & 197 & 199 & 7 \\
Total & 4 & 253 & 257 & 20 \\
\hline
\end{tabular}

Note: Chi-squared $=1.75 ; P=0.19$.

Abbreviation: G-FOBT, guaiac fecal occult blood test.

difference in negative predictive values for both methods, being 98.99\% (95\% CI $=96.42-99.88)$ and 99.56\% (95\% CI =97.59-99.99), respectively.

Table 5 shows the sensitivity of FOB by FIT, which was significantly higher for stages III and IV CRC than for stages I and II $(P=0.01)$ and insignificant for G-FOBT $(P=0.07)$.

\section{Discussion}

We compared the sensitivity and specificity of a guaiac test and a monoclonal antibody-based immunoassay performed on 277 older patients reporting to the King Fahd Military Medical Complex and King Faisal Specialist Hospital for the detection of occult blood and the prediction of CRC in a hospital setting.

In this study, the occult blood positivity rates of the same samples were $22.6 \%$ and $12.1 \%$ by guaiac and immunoassay tests, respectively. The high incidence of false-positive test results by guaiac-based testing is largely due to the lack of proper dietary restrictions for several days prior to testing. In another study, even with properly prepared patients, the incidence of false-positive results with G-FOBT was found to be as high as $10 \% .{ }^{26}$ Red meat, fruits, and vegetables high in peroxidase, high doses of ascorbic acid (vitamin $\mathrm{C}$, $250 \mathrm{mg}$ /day or more), oral medications such as aspirin or other nonsteroidal anti-inflammatory drugs, heavy alcohol consumption, and some others may interfere with the test. $^{27}$

Table 3 Performance characteristics of FIT for detecting colorectal cancer

\begin{tabular}{|c|c|c|c|c|}
\hline \multirow[t]{3}{*}{ FIT } & \multirow{2}{*}{\multicolumn{2}{|c|}{$\frac{\text { Screening group }}{\text { Colorectal cancer }}$}} & \multirow[t]{3}{*}{ Total } & \multirow{3}{*}{$\frac{\text { Control group }}{\text { Colorectal cancer }}$} \\
\hline & & & & \\
\hline & Positive & Negative & & \\
\hline Positive & 3 & 25 & 28 & 16 \\
\hline Negative & I & 228 & 229 & 4 \\
\hline Total & 4 & 253 & 257 & 20 \\
\hline
\end{tabular}

Note: Chi-squared $=17.20 ; P=0.00$.

Abbreviation: FIT, fecal immunochemical test.
Table 4 Sensitivity, specificity, and predictive values and $95 \% \mathrm{Cl}$ (between parenthesis) of G-FOBT and FIT for colorectal cancer $(n=277)$

\begin{tabular}{lll}
\hline & G-FOBT & FIT \\
\hline Sensitivity & $50.00 \%(6.76-93.24)$ & $75.00 \%(\mid 9.4 I-99.37)$ \\
Specificity & $77.87 \%(72.24-82.83)$ & $90.12 \%(85.76-93.50)$ \\
Positive likelihood ratio & $2.26(0.83-6.18)$ & $7.59(3.86-14.94)$ \\
Negative likelihood ratio & $0.64(0.24-I .7 I)$ & $0.28(0.05-I .52)$ \\
Positive predictive value & $3.45 \%(0.42-I I .9 I)$ & $10.7 I \%(2.27-28.23)$ \\
Negative predictive value & $98.99 \%(96.42-99.88)$ & $99.56 \%(97.59-99.99)$ \\
\hline
\end{tabular}

Abbreviations: $\mathrm{Cl}$, confidence interval; G-FOBT, guaiac fecal occult blood test; FIT, fecal immunochemical test; $n$, number.

The number of observed agreements between the two test methods is $240(86.6 \%)$, whereas $37(13.4 \%)$ samples showed a discrepant result between the two methods. Because there is no "gold standard" reference method for FOBT, we could not resolve which method gave the "correct" results among the discrepant cases.

On the other hand, FIT methods are specific for human hemoglobin and require no dietary preparation, although medications that may cause minor gastrointestinal bleeding will potentially produce positive results. In normal subjects, a small amount of blood is lost in the intestine each day. It has been stated before that a blood loss of 2-3 mL (approximately $0.3 \mathrm{mg}$ hemoglobin/g of stool) is the lower limit of blood loss that may be associated with gastrointestinal pathology. ${ }^{28}$ It has been indicated before that performing two FOBT tests does not improve diagnostic accuracy; rather, it increases costs. ${ }^{29}$

Unlike many previous studies, ${ }^{17,19,22}$ the colonoscopy results were also available for all those that were tested for occult blood, including those with negative results, which allowed for the improved detection of specificities. The sensitivity of G-FOBT and FIT for the detection of CRC was 50.00\% (95\% CI $=6.76-93.24)$ and $75.00 \%(95 \% \mathrm{CI}$ $=19.41-99.37)$, respectively. The specificity of G-FOBT and FIT was $77.87 \%(95 \% \mathrm{CI}=72.24-82.83)$ and $90.12 \%(95 \%$ $\mathrm{CI}=85.76-93.50)$, respectively.

A number of studies have reported clinical evaluations of various FOBT methods, and guidelines have been published for CRC screening with varying levels of sensitivities and specificities for detecting CRC, depending on the study design. Generally, the sensitivity of FOBT for CRC is relatively low, between $30 \%$ and $80 \%$, in most population-based studies, ${ }^{30-33}$ while specificity has been reported to be between $87 \%$ and $98 \% .^{32}$ In agreement with our findings, a systematic review of the literature on repeated annual or biennial ColoScreen or G-FOBT revealed that the reported sensitivity 
Table 5 The sensitivities and $95 \% \mathrm{Cl}$ (between parentheses) of a single G-FOBT and FIT by different anatomic stages of colorectal cancer

\begin{tabular}{llllll}
\hline & $\mathbf{n}$ & $\begin{array}{l}\text { G-FOBT } \\
\text { positive cases }\end{array}$ & $\begin{array}{l}\text { G-FOBT sensitivity \% } \\
(\mathbf{9 5 \%} \mathbf{C l})\end{array}$ & $\begin{array}{l}\text { FIT } \\
\text { positive cases }\end{array}$ & $\begin{array}{l}\text { FIT sensitivity \% } \\
(\mathbf{9 5 \%} \mathbf{C l})\end{array}$ \\
\hline Stage I & 2 & $\mathrm{I}$ & $50.0(\mathrm{I} .26-98.74)$ & $\mathrm{I}$ & $50.0(1.26-98.74)$ \\
Stage II & 15 & 8 & $53.33(26.59-78.73)$ & 9 & $60.0(32.29-83.66)$ \\
Stage III & 3 & 2 & $66.67(9.43-99.16)$ & 2 & $66.67(9.43-99.16)$ \\
Stage IV & 3 & 2 & $66.67(9.43-99.16)$ & 3 & $100.00(29.24-100.00)$ \\
$P$-value & & $t=3.68 ; P=0.066$ & & $t=2.83 ; P=0.005$ & \\
\hline
\end{tabular}

Abbreviations: $\mathrm{Cl}$, confidence interval; G-FOBT, guaiac fecal occult blood test; FIT, fecal immunochemical test; $n$, number.

for CRC varied from $51 \%$ to $100 \%$, and specificity varied from $90 \%$ to $97 \% .{ }^{26}$ Furthermore, the positive predictive value (the percentage of positive tests that are true-positive results) ranged from $2.4 \%$ to $17.0 \% .{ }^{33}$ Low sensitivity for detecting $\mathrm{CRC}$ using guaiac-based tests was also reported in a population-based study. ${ }^{17,34}$ Immunochemical tests for FOBT have been studied as an alternative to guaiac-based tests. ${ }^{35}$

Many studies have shown that FIT has better performance characteristics when compared to most G-FOBTs, and it also detects advanced adenomas, with a sensitivity for advanced adenomas ranging from $20 \%$ to $40 \% .{ }^{17-19,36}$ FIT testing has been shown to facilitate compliance and it improves specificity for CRC screening, but at an increased cost compared to guaiac-based FOBT ${ }^{37}$ FITs are moderately sensitive, highly specific, and they have high overall diagnostic accuracy for detecting CRC..$^{38}$ However, different brands of quantitative FITs, even those using the same cutoff hemoglobin concentration, perform differently in mass screening. ${ }^{39}$ Comparisons of different techniques to detect occult blood in stool have been widely performed.

This study, which is in agreement with some others, ${ }^{40-42}$ indicated the higher sensitivity, specificity, and predictive values of FIT for the screening of CRC when compared to community-based studies. ${ }^{43}$

We detected only a few cases of CRCs among the screening group that resulted in a wide range of $95 \%$ CIs for sensitivity. However, specificity was well detected with a high degree of confidence. Moreover, the positive likelihood ratio of FIT for the detection of CRC was 7.59 (95\% $\mathrm{CI}=3.86-14.94)$, indicating good performance of the test.

When classifying the tumors according to the different stages, the sensitivity of FOB by FIT was significantly higher for stages III and IV CRC than for stages I and II, which is in agreement to what was reported before. ${ }^{44}$

\section{Conclusion}

We believe that FIT may offer advantages over G-FOBT in hospital settings because its higher sensitivity, combined with its low incidence of false-positive test results, could improve screening programs for CRC.

It is worth mentioning that our sample size was only modest, we had a limited follow-up period, and the FOBT was administered once.

\section{Acknowledgments}

This work was supported by the Prince Sultan Military College of Health Sciences. We thank Mr Abdulla Al Shehri, who helped us a lot with the procurement of supplies and samples, and $\mathrm{Mr}$ Mohi Hussein for his great help in the laboratory work.

\section{Disclosure}

The authors report no conflicts of interest in this work.

\section{References}

1. Mosli MH, Al-Ahwal MS. Colorectal cancer in the Kingdom of Saudi Arabia: need for screening. Asian Pac J Cancer Prev. 2012;13(8): 3809-3813.

2. Ministry of Health. Cancer Incidence Report Saudi Arabia 2003 Riyadh, Saudi Arabia: Kingdom of Saudi Arabia, Ministry of Health, National Cancer Registry; 2003.

3. Ibrahim EM, Zeeneldin AA, El-Khodary TR, Al-Gahmi AM, Bin Sadiq BM. Past, present and future of colorectal cancer in the Kingdom of Saudi Arabia. Saudi J Gastroenterol. 2008;14(4): $178-182$.

4. McLoughlin RM, O'Morain CA. Colorectal cancer screening. World J Gastroenterol. 2006;12(42):6747-6750.

5. Zavoral M, Suchanek S, Zavada F, et al. Colorectal cancer screening in Europe. World J Gastroenterol. 2009;15(47):5907-5915.

6. Ostrow JD. Tests for fecal occult blood. In: Walker HK, Hall WD, Hurst JW, editors. Clinical Methods: The History, Physical and Laboratory Examinations. 3rd ed. Boston, MA: Butterworths; 1990. Chapter 98:489-491.

7. Brevinge H, Lindholm E, Buntzen S, Kewenter J. Screening for colorectal neoplasia with faecal occult blood testing compared with flexible sigmoidoscopy directly in a 55-56 years' old population. Int J Colorectal Dis. 1997;12(5):291-295.

8. Hardcastle JD, Chamberlain JO, Robinson MH, et al. Randomised controlled trial of faecal-occult-blood screening for colorectal cancer. Lancet. 1996;348(9040):1472-1477.

9. Kronborg O, Fenger C, Olsen J, Jørgensen OD, Søndergaard O. Randomised study of screening for colorectal cancer with faecal-occultblood test. Lancet. 1996;348(9040):1467-1471.

10. Kratochvil JF, Burris RH, Seikel MK, Harkin JM. Isolation and characterization of $\alpha$-guaiaconic acid and the nature of guaiacum blue. Phytochemistry. 1971;10:2529-2531. 
11. Scriven AJ, Tapley EM. Coloscreen VPI test kit evaluated for detection of fecal occult blood. Clin Chem. 1989;35(1):156-158.

12. Young GP. Cole S. New stool screening tests for colorectal cancer. Digestion. 2007;76(1):26-33.

13. Young Gp, St John DJB. Faecal occult blood tests: choice, usage and clinical applications. Clin Biochem Rev. 1992;13:161-167.

14. Greegor DH. A progress report. Detection of colorectal cancer using guaiac slides. CA Cancer J Clin. 1972;22(6):360-363.

15. Mandel JS, Church TR, Bond JH, et al. The effect of fecal occultblood screening on the incidence of colorectal cancer. $N$ Engl J Med. 2000;343(22):1603-1607.

16. Allison JE. Review article: faecal occult blood testing for colorectal cancer. Aliment Pharmacol Ther. 1998;12(1):1-10.

17. van Rossum LG, van Rijn AF, Laheij RJ, et al. Random comparison of guaiac and immunochemical fecal occult blood tests for colorectal cancer in a screening population. Gastroenterology. 2008;135(1): $82-90$.

18. Freitas BRV, Nagasako CK, Pavan CR, et al. Immunochemical fecal occult blood test for detection of advanced colonic adenomas and colorectal cancer: comparison with colonoscopy results. Gastroenterol Res Prac. 2013.

19. Dancourt V, Lejeune C, Lepage C, Gailliard MC, Meny B, Faivre J. Immunochemical faecal occult blood tests are superior to guaiacbased tests for the detection of colorectal neoplasms. Eur J Cancer. 2008;44(15):2254-2258.

20. Akhtar R, Lee M, Itzkowitz SH. Colonoscopy versus computed tomography colonography for colorectal cancer screening. Mt Sinai J Med. 2010;77(2):214-224.

21. Allison JE, Fraser CG, Halloran SP, Young GP. Population Screening for Colorectal Cancer Means Getting FIT: The Past, Present, and Future of Colorectal Cancer Screening Using the Fecal Immunochemical Test for Hemoglobin (FIT). Gut Liver. 2014 Mar; 8(2): 117-130.

22. Guittet L, Bouvier V, Mariotte N, et al. Comparison of a guaiac based and an immunochemical faecal occult blood test in screening for colorectal cancer in a general average risk population. Gut. 2007; 56(2):210-214.

23. Bossuyt PM, Irwig L, Craig J, Glasziou P. Comparative accuracy: assessing new tests against existing diagnostic pathways. $\mathrm{Br}$ Med $\mathrm{J}$ 2006; 332:1089-9102.

24. American Joint Committee on Cancer. AJCC Cancer Staging Manual. 7th ed. New York, NY: Springer, 2010.

25. Burch JA, Soares-Weiser K, St John DJ, et al. Diagnostic accuracy of faecal occult blood tests used in screening for colorectal cancer: a systematic review. J Med Screen. 2007;14(3):132-137.

26. Macrae FA, St John DJ. Relationship between patterns of bleeding and hemoccult sensitivity in patients with colorectal cancers or adenomas. Gastroenterology. 1982;82(5 Pt 1):891-898.

27. Hernandez V, Cubiella J, Gonzalez-Mao MC, et al; COLONPREV Study Investigators. Fecal immunochemical test accuracy in average-risk colorectal cancer screening. World J Gastroenterol. 2014;20(4):1038-1047.

28. Hewitson P, Glasziou P, Watson E, Towler B, Irwig L. Cochrane systematic review of colorectal cancer screening using the fecal occult blood test (hemoccult): an update. Am J Gastroenterol. 2008; 103(6):1541-1549.

29. Whitlock EP, Lin JS, Liles E, Beil TL, Fu R. Screening for colorectal cancer: a targeted, updated systematic review for the US Preventive Services Task Force. Ann Intern Med. 2008;149(9):638-658.
30. Ekelund G, Manjer J, Zackrisson S. Population-based screening for colorectal cancer with faecal occult blood test - do we really have enough evidence? Int J Colorectal Dis. 2010;25(11):1269-1275.

31. Rabeneck L, Zwaal C, Goodman JH, Mai V, Zamkanei M. Cancer Care Ontario guaiac fecal occult blood test (FOBT) laboratory standards: evidentiary base and recommendations. Clin Biochem. 2008; 41(16-17):1289-1305.

32. Levi Z, Rozen P, Hazazi R, et al. A quantitative immunochemical fecal occult blood test for colorectal neoplasia. Ann Intern Med. 2007; 146(4):244-255.

33. Wong CK, Fedorak RN, Prosser CI, Stewart ME, van Zanten SV, Sadowski DC. The sensitivity and specificity of guaiac and immunochemical fecal occult blood tests for the detection of advanced colonic adenomas and cancer. Int J Colorectal Dis. 2012;27(12):1657-1664.

34. Hundt $\mathrm{S}$, Haug U, Brenner H. Comparative evaluation of immunochemical fecal occult blood tests for colorectal adenoma detection. Ann Intern Med. 2009;150(3):162-169.

35. Levin B, Lieberman DA, McFarland B, et al; American Cancer Society Colorectal Cancer Advisory Group; US Multi-Society Task Force; American College of Radiology Colon Cancer Committee. Screening and surveillance for the early detection of colorectal cancer and adenomatous polyps, 2008: a joint guideline from the American Cancer Society, the US Multi-Society Task Force on Colorectal Cancer, and the American College of Radiology. CA Cancer J Clin. 2008; 58(3):130-160.

36. Smith A, Young GP, Cole SR, Bampton P. Comparison of a brushsampling fecal immunochemical test for hemoglobin with a sensitive guaiac-based fecal occult blood test in detection of colorectal neoplasia. Cancer. 2006;107(9):2152-2159.

37. Brenner H, Tao S, Haug U. Low-dose aspirin use and performance of immunochemical fecal occult blood tests. JAMA. 2010; 304(22):2513-2520.

38. Lee JK, Liles EG, Bent S, Levin TR, Corley DA. Accuracy of fecal immunochemical tests for colorectal cancer: systematic review and meta-analysis. Ann Intern Med. 2014;160(3):171.

39. Chiang TH, Chuang SL, Chen SL, et al. Difference in performance of fecal immunochemical tests with the same hemoglobin cutoff concentration in a nationwide colorectal cancer screening program. Gastroenterology. 2014;147(6):1317-1326.

40. Chiang TH, Lee YC, Tu CH, Chiu HM, Wu MS. Performance of the immunochemical fecal occult blood test in predicting lesions in the lower gastrointestinal tract. CMAJ. 2011;183(13):1474-1481.

41. Crotta S, Castiglione G, Grazzini G, Valle F, Mosconi S, Rosset R. Feasibility study of colorectal cancer screening by immunochemical faecal occult blood testing: results in a northern Italian community. Eur J Gastroenterol Hepatol. 2004;16(1):33-37.

42. Grazzini G, Castiglione G, Ciabattoni C, et al. Colorectal cancer screening programme by faecal occult blood test in Tuscany: first round results. Eur J Cancer Prev. 2004;13(1):19-26.

43. Hol L, van Leerdam ME, van Ballegooijen M, et al. Screening for colorectal cancer: randomised trial comparing guaiac-based and immunochemical faecal occult blood testing and flexible sigmoidoscopy. Gut. 2010;59(1):62-68.

44. Sohn DK, Jeong SY, Choi HS, et al. Single immunochemical fecal occult blood test for detection of colorectal neoplasia. Cancer Res Treat. 2005;37(1):20-23.
Clinical and Experimental Gastroenterology

\section{Publish your work in this journal}

Clinical and Experimental Gastroenterology is an international, peerreviewed, open access journal, publishing all aspects of gastroenterology in the clinic and laboratory, including: Pathology, pathophysiology of gastrointestinal disease; Investigation and treatment of gastointestinal disease; Pharmacology of drugs used in the alimentary tract;

\section{Dovepress}

Immunology/genetics/genomics related to gastrointestinal disease. This journal is indexed on CAS. The manuscript management system is completely online and includes a very quick and fair peer-review system. Visit http://www.dovepress.com/testimonials.php to read real quotes from published authors. 